\title{
Luminosity Dependent Changes of Pulse Profiles in the X-ray Binary GX $1+4$
}

\author{
J. G. Greenhill ${ }^{1}$, D. Galloway ${ }^{1,2}$ and M. C. Storey ${ }^{2}$ \\ 1 Physics Department, University of Tasmania, GPO Box 252C, \\ Hobart, Tas. 7001, Australia \\ John.Greenhill@utas.edu.au, Duncan.Galloway@utas.edu.au \\ 2 Special Research Centre for Theoretical Astrophysics, School of Physics, \\ University of Sydney, NSW 2006, Australia \\ michelle@physics.usyd.edu.au \\ Received 1998 March 4, accepted 1998 April 28
}

\begin{abstract}
We have reviewed the X-ray pulse profiles from a large number of observations of the accreting binary pulsar GX $1+4$ obtained during the last 25 years. The profiles cover various energy ranges between 1 and $100 \mathrm{keV}$. Using these data we present a coherent picture of present and past pulse profiles and the variations of these pulse profiles with time. The pulse shape is dependent on both the X-ray luminosity and whether the pulsar is spinning up or down. Profiles measured during the GX $1+4$ high state in the 1970s are all trailing edge bright. Subsequently the profiles have generally been symmetric or leading edge bright. Rossi X-ray Timing Explorer (RXTE) satellite data taken in July 1996 show that similar pulse shape variations can occur on a timescale of hours. The implications of this new information for accretion models is discussed.
\end{abstract}

Keywords: X-rays: stars — accretion disks — pulsars: individual: GX $1+4$

\section{Introduction}

The variable X-ray source GX $1+4$ associated with the M6III class giant V2116 Ophiuchi has exhibited a variety of interesting behaviour throughout the history of observations. The extended X-ray bright state of the 1970s, during which came the first detection (Lewin et al. 1971), was followed by a period of non-detection during the early 1980s and then a low intensity state which has persisted to this day. The pulse period is $\sim 2$ minutes. Average spin-up during the 1970s gave way to spin-down after the period of non-detection, although brief periods of spin-up have been detected recently (Chakrabarty et al. 1997). The measured rates of spin-up and spindown ( $\sim 2 \%$ per annum) are similar in magnitude and are among the fastest known for any pulsar.

A large set of pulse profiles taken at different times and in different intensity states now exists. Because of their strong energy dependence and great variability, relative phasing between observations at different times and energies is not clear. In this paper we report the results of a study of these published profiles using the wide energy range observations of White et al. (1983) to determine their relative phases. We show evidence of an association between X-ray luminosity $\left(L_{X}\right)$, the spin-up/spin-down state and the pulse shape.

\section{General Features of GX 1+4 Pulse Profiles}

Pulse profiles obtained from GX $1+4$ can be broadly classified into two classes which have generally been found during the two distinct eras - the 1970s high intensity spin-up era and the later low intensity, principally spin-down, era. These are illustrated in Figures 1 and 2 respectively. The low energy profiles in both classes have a characteristic narrow 'dip' or primary minimum. The width corresponds to only $5 \%-10 \%$ of the period and is not always obvious in data from the 1970s when sampling rates were relatively low. We define the bottom of the 'dip' as phase zero for the system. At other phases the profiles can be complex; particularly at low energies. At higher energies the profile is generally simpler and the phase of deepest minimum corresponds to that of the low energy 'dip' (Doty et al. 1981; White et al. 1983). A secondary minimum is sometimes present at phase $\sim 0.5$ (e.g. Doty et al. 1981; Makishima et al. 1988; Greenhill et al. 1989; Paul et al. 1997).

Other significant features are sometimes observed in the GX 1+4 pulse profiles. Greenhill et al. (1993) observed a remarkable change in pulse shape at energies above $60 \mathrm{keV}$. A narrow emission 'spike' appeared at the phase $(0.5)$ of the lower energy secondary minimum. Dotani et al. (1989) observed 
a very narrow emission 'spike' at the position of primary minimum (phase zero) in $1-7 \mathrm{keV}$ data. White et al. (1983) observed a phase reversal with the low energy 'notch' $(1-7 \mathrm{keV})$ being replaced at higher energies by an emission peak. Considerable variation between successive individual pulses was reported by Elsner et al. (1985).

(a)

(b)

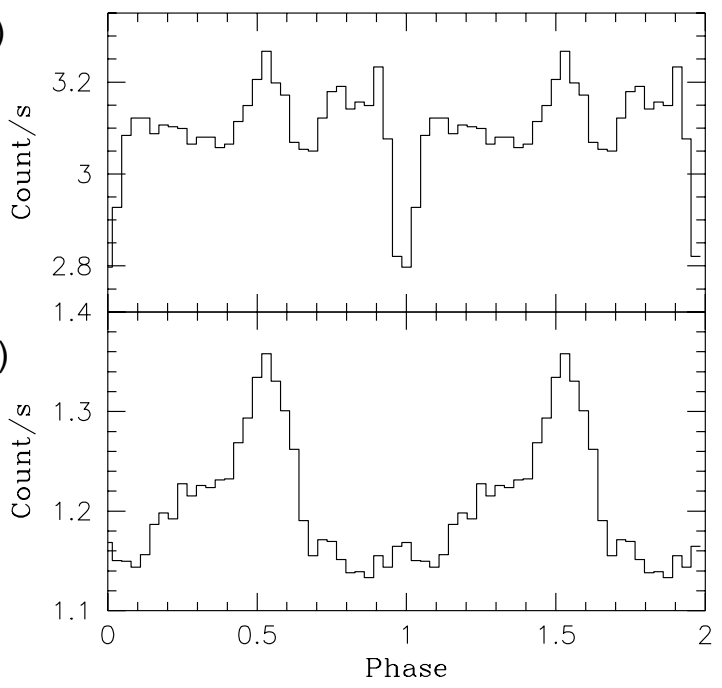

Figure 1-Typical pre-1980 (type A) pulse profiles from GX 1+4: (a) low $(1 \cdot 5-3 \mathrm{keV})$ energy band and (b) the high $(30-40 \mathrm{keV})$ band (Doty et al. 1981). The $1 \sigma$ error limits are $0.6 \%$ and $1.0 \%$ respectively. The phasing has been changed to suit the definition in Section 2.

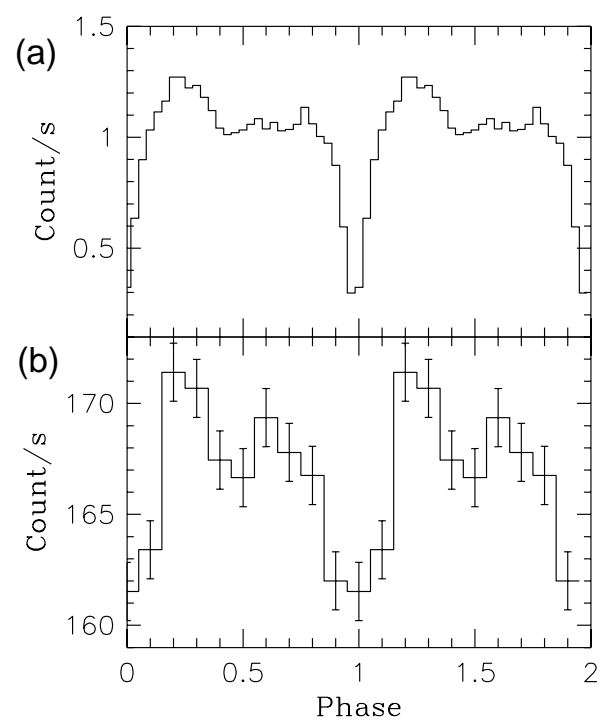

Figure 2-Post-1980 (type B) pulse profiles in (a) the low energy (0.5-10 keV) ASCA data (Kotani et al. 1997) and (b) the high energy (20-75 keV) band (Greenhill et al. 1993). The error limits in the ASCA data are too small to display. The phasing has been changed to suit the definition in Section 2.

The emission peak is frequently asymmetric, particularly at higher energies. This can be used to classify the profiles into two classes. Class A profiles (Figure 1) have the trailing edge (i.e. the section of pulse profile at the highest phase) brightestusing our definition of phase 0 . These are found, almost exclusively, in data obtained during the high intensity state. Typical examples are in Doty et al. (1981), White et al. (1983) and Elsner et al. (1985). During the low intensity, mostly spin-down state, since 1986, the pulses are generally either symmetric or have the opposite asymmetry with the leading edge brightest, i.e. they have the section of pulse profile at lower phases brightest. We designate these class B. Examples are in Makashima et al. (1988), Greenhill et al. (1989), Mony et al. (1991) and Storey et al. (1998, present issue p. 217).

Classification into two classes is somewhat arbitrary since many profiles are almost symmetric. The shape appears to depend in a complex way upon X-ray luminosity and whether the pulsar is spinning up or down. We know of no examples of class B profiles being observed during the high state in the 1970s when spin-up appears to have been almost continuous. Since 1986, when the luminosity was generally lower and occasional brief episodes of spin-up have punctuated an average spin-down of the pulsar, the profiles have mostly been symmetric or class B.

Class A profiles have, however, been observed on several occasions. During RXTE observations lasting 34 hours on 19-21 July 1996 the profiles changed from class B to class A (Giles et al. 1998). This occurred at all energies from $2-60 \mathrm{keV}$. The change occurred after a short interval when the intensity became very low $\left(<2 \times 10^{-13} \mathrm{~W} \mathrm{~m}^{-2}\right.$ in the $10-60 \mathrm{keV}$ energy range). At about the same time as the RXTE measurements the BATSE experiment on GRO detected a brief X-ray flare and transition from pulsar spin-down to spin-up (Chakrabarty et al. 1997). A detailed analysis of this event will be published elsewhere (Giles et al. 1998). In March 1991 Laurent et al. (1993) recorded class A profiles during high energy (40$77 \mathrm{keV}$ ) measurements with the SIGMA experiment on GRANAT. We note, however, that the X-ray intensity during the SIGMA measurements was at a level comparable to that during the short-lived BATSE spin-up episode in 1996. BATSE was not operational at this time and it is possible that a short term spin-up event was under way during the SIGMA measurements. In October 1991, when the X-ray luminosity was slightly lower, the profiles were symmetric (Laurent et al. 1993). Similarly, in September 1990 Kotani et al. (1997) detected class A (trailing edge bright) profiles during Ginga satellite measurements. The X-ray luminosity was relatively high at the time. A year later the luminosity was much lower and the profiles were symmetric.

We have investigated the dependence of the asymmetry upon X-ray luminosity using a number of published high energy $(E>20 \mathrm{keV})$ profiles 
for which the phase of primary minimum could be identified and for which spectral intensities are available. We use only the high energy data since the complexity of the low energy profiles makes it difficult to quantify their asymmetry. Furthermore, published profiles are not in general strongly dependent on energy above $20 \mathrm{keV}$. Hence valid comparisons can be made between data sets covering somewhat different energy ranges. The intensity at $20 \mathrm{keV}$ is taken as an overall indicator of X-ray luminosity $L_{X}$. This was estimated in some cases by extrapolation from higher energies assuming a power law spectrum with a photon index of 2 .

We define an asymmetry parameter $\alpha$ as the ratio of the pulsed flux between phases 0.5 and 0.75 to the pulsed flux between phases $0 \cdot 25$ and $0 \cdot 5$. Hence class A profiles have $\alpha>1$ and class B have $\alpha \leq 1$. In the absence of a phase link between published observations we assume that the phase of primary minimum is phase zero. In some data sets (e.g. White et al. 1983) the minimum is broad with indications of a possible weak emission feature with phase width $\sim 0 \cdot 2$ superimposed on the minimum. In these cases we have assigned phase 0 to the minimum of a smoothed curve fit to the principal modulation. This causes considerable uncertainty in the time of phase 0 , but is justified by the close agreement with the time of the low energy 'dip' in the data sets where this was known.

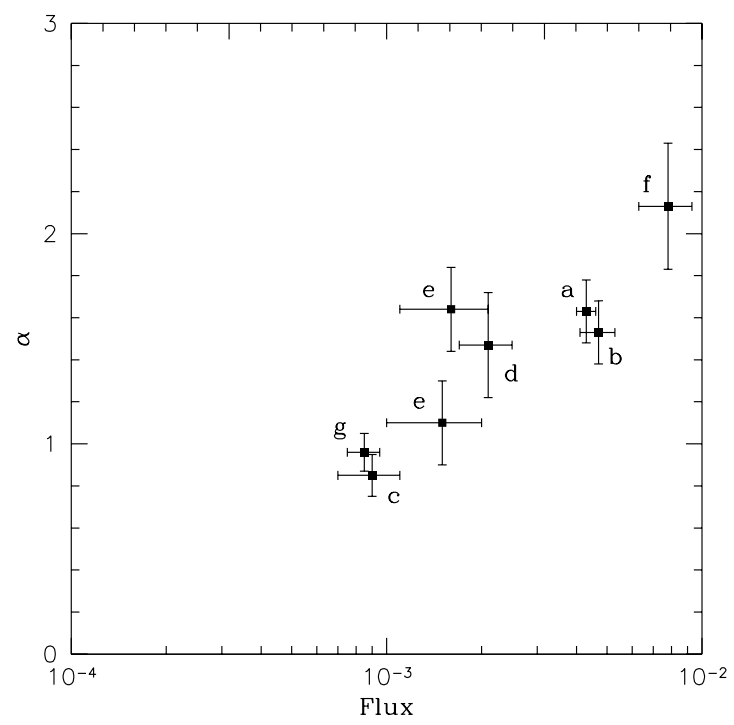

Figure 3-Asymmetry parameter $\alpha$ vs $20 \mathrm{keV}$ intensity $\left(\mathrm{cm}^{-2} \mathrm{~s}^{-1} \mathrm{keV}^{-1}\right)$ for published hard X-ray (>20 keV) measurements: (a) White et al. (1983), (b) Doty et al. (1981), (c) Greenhill et al. (1993), (d) Kendziorra et al. (1982), (e) Laurent et al. (1993), (f) Maurer et al. (1982) and (g) Mony et al. (1991).

The relationship between $\alpha$ and $L_{X}$ is illustrated in Figure 3. It seems likely that a correlation exists although more data points are required to verify this. The form of the relationship depends strongly on the choice of phase 0 and this is poorly defined.
Clearly large $\alpha$ profiles were observed when $L_{X}$ was high in the 1970s and $\alpha$ has been smaller since the mid 1980s when $L_{X}$ was generally low. This does not, however, prove a direct causal relationship between $\alpha$ and $L_{X}$. The RXTE measurements by Giles et al. (1998) suggest that $\alpha$ depends also on whether the pulsar is spinning up or down. The transition from class A to class B profiles (small $\alpha$ to large $\alpha$ ) occurred when $L_{X}$ was very low. We note however that the transition was followed by a large increase in $L_{X}$ and a brief resumption of spin-up (Chakrabarty et al. 1997). We suggest therefore that the change in profile was a consequence of a transition in the accretion flow which led to the resumption of spin-up and subsequent large increase in $L_{X}$. A similar brief episode of spin-up probably occurred at about the time of the SIGMA measurements in March 1991 (Laurent et al. 1993).

\section{Discussion}

The explanation for the pulse profile changes is not clear. It seems reasonable to assume that the changes are associated with a change in the accretion flow around the neutron star. Asymmetries in pulse profiles can result from magnetic field asymmetry, caused for example by a non-centred dipole (e.g. Padden and Storey 1986; Kraus et al. 1995), or higher-order multipole field components. In the case of an asymmetric magnetic field distribution the varying strength of the magnetic field sweeping by the accretion disk with phase leads to different rates of accretion onto different regions of the polar cap. Different stable states of accretion could lead to different pulse shapes and different spin-up/spindown rates.

Asymmetries can also arise because of the relative rotation of the neutron star and the incoming matter. The connection between pulse shape and the relative rotation of the accretion plasma and star has been made previously by Wang and Welter (1981). We have demonstrated a correlation between pulse shape and X-ray luminosity and a linkage between this and the spin-up/spin-down behaviour of GX $1+4$. This is consistent with the work of Wang \& Welter (1981) who showed that class A or class B pulse profiles can be produced depending on whether the specific angular momentum of the accreting plasma is greater than or less than that of the neutron star. If the specific angular momentum of the accreting plasma is greater than that of the neutron star, the trailing edge of pulse profiles will be brighter (Wang \& Welter 1981). If the plasma specific angular momentum is less than that of the star the leading edge of the pulse profile will be brighter.

There has been considerable uncertainty as to whether the deep primary minimum observed in most pulse profiles from $\mathrm{GX} 1+4$ is the pulse centre, i.e. the closest passage of the line-of-sight to the 
magnetic pole of the star where the emission region is located, or whether the secondary minimum sometimes observed is the pulse centre (see, for example, Storey, Greenhill \& Kotani 1998). The information above can be used to help determine which section of the pulse profile in GX $1+4$ represents the pulse centre. If the pulse asymmetry is caused by differential rotation of the accretion plasma and the neutron star, then during the spinup era of the 1970s (when the accretion plasma specific angular momentum was presumably more than the star specific angular momentum) one would expect trailing-edge bright pulses. An examination of observed pulse profiles from the 1970s shows that the section of pulse immediately before the deep minimum is the brightest. Therefore, from the above analysis, one concludes that the section of pulse immediately before the deep minimum is the trailing edge of the pulse implying that the deep minimum is the edge of the pulse, not the pulse centre. Similarly, during the spin-down era of the 1980s (when the star specific angular momentum was presumably more than that of the accretion plasma) one would expect leading-edge-bright pulses. An examination of observed pulse profiles from the spin-down era shows that the brightest part of the pulse is the part immediately following the deep minimum. Using the analysis of Wang \& Welter (1981) this part of the pulse is the leading part of the pulse, which again indicates that the primary minimum is the edge of the pulse.

Evidence has emerged recently that in the spindown state, at least on some occasions, a retrograde accretion disk is forming in $\mathrm{GX} 1+4$. In the standard theory of disk accreting X-ray binaries, assuming a disk rotating in the same direction as the star (Ghosh \& Lamb 1979), spin-down is thought to occur when the accretion rate and thus the ram pressure of the flow drops to the point where the inner edge of the accretion disk is pushed outwards by magnetic pressure to a radius where the specific angular momentum of accreting matter is less than that of the neutron star. In this theory one expects that an increase in the spin-down rate would be associated with a decrease in luminosity. However, recently Nelson et al. (1997) have found that GX 1+4 spun down faster during a brief X-ray flare and they point out that this observation could be explained if GX $1+4$ had a retrograde accretion disk during the flare, consistent with an earlier suggestion that the accretion disk in GX $1+4$ is alternating between retrograde and prograde rotation (Makishima et al. 1988).

Cui (1997) has presented observational evidence for the effect mentioned above where a decrease in accretion rate leads to the inner edge of the accretion disk being pushed outwards from the star until accretion is halted and pulsation stops. Cui derived from the observational results an estimate for the surface magnetic field strength for GX $1+4$ of $3 \cdot 1 \times 10^{13} \mathrm{G}\left(3 \cdot 1 \times 10^{9} \mathrm{~T}\right)$, accurate to within a factor of two. This estimate is consistent with earlier estimates for the surface field strength based on emission mechanism modelling and pulse phase spectroscopy (Greenhill et al. 1993) and by assuming the formation of a retrograde accretion disc in GX 1+4 (Dotani et al. 1989).

Our demonstration of a correlation between pulse shape and luminosity and the relation to spinup/spin-down behaviour gives us a new tool with which to explore the accretion disk physics in this, and possibly other, systems. The RXTE results to be presented elsewhere (Giles et al. 1998) show the transition from leading-edge profile to trailing edge profile occurring over a short time period. The fact that the profile shape changed before the rise in luminosity is consistent with the hypothesis that there was an accompanying transition in disk rotation rate. A full analysis of the 1996 RXTE observations of GX $1+4$ (Giles et al. 1998) should shed further light on the pulse morphology of GX $1+4$.

\section{Acknowledgments}

We thank Barry Giles for making RXTE data available to us prior to its publication.

Chakrabarty, D., Bildsten, L., Grunsfeld, J. M., Koh, D. T., Prince, T. A., \& Vaughan, B. A. 1997, ApJ, 474, 414

Cui, W. 1997, ApJ, 482, L163

Dotani, T., Kii, T., Nagase, F., Makishima, K., Ohashi, T., Sakao, T., Koyama, K., \& Tuohy, I. R. 1989, PASJ, 41, 427

Doty, J. P., Hoffman, J. A., \& Lewin, W. H. G. 1981, ApJ, 243, 257

Elsner, R. F., Weisskopf, M. C., Apparao, K. M. V., Darbro, W., Ramsey, B. D., Williams, A. C., Grindlay, J. E., \& Sutherland, P. G. 1985, ApJ, 297, 288

Ghosh, P., \& Lamb, F. K. 1979, ApJ, 234, 296

Giles, A. B., Galloway, D. K., Greenhill, J. G., Storey, M. C., \& Swank, J. H. 1998, in preparation

Greenhill, J. G., Giles, A. B., Sharma, D. P., Dieters, S., Sood, R. K., Thomas, J. A., Waldron, L., Manchanda, R. K., Carli, R., Hammer, P., Kendziorra, E., Staubert, R., Bazzano, A., Ubertini, P., \& La Padula, C. 1989, A\&A, 208, L1

Greenhill, J. G., Sharma, D. P., Dieters, S. W. B., Sood, R. K., Waldron, L., \& Storey, M. C. 1993, MNRAS, 260, 21

Kendziorra, E., Staubert, R., Reppin, C., Pietsch, W., Voges, W., \& Trümper, J. 1982, in Galactic X-ray Sources, ed. P. Sanford et al. (New York: Wiley), p. 205

Kotani, T., Dotani, T., Nagase, F., Greenhill, J., Pravdo, S. H., \& Angelini, L. 1997, preprint

Kraus, U., Nollert, H. P., Ruder, H., \& Riffert, H. 1995, ApJ, 450, 763

Laurent, P., Salotti, L., Paul, J., Lebrun, F., Denis, M., Barret, D., Jourdain, E., Roques, J. P., Churazov, E., Gilfavov, M., Sunyaev, R., Diachkov, N., Khavenson, N., Novikov, B., Chulkov, I., \& Kuznetzov, A. 1993, A\&A, 278,444 
Lewin, W. H. G., Ricker, G. R., \& McClintock, J. E. 1971, ApJ, 169, L17

Makishima, K., Ohashi, T., Sakao, T., Dotani, T., Inoue, H., Koyama, K., Makino, F., Mitsuda, K., Nagase, F., Thomas, H. D., Turner, M. J. L., Kii, T., \& Tawara, Y. 1988, Nature, 333, 746

Maurer, G. S., Johnson, W. N., Kurfess, J. D., \& Strickman, M. S. 1982, ApJ, 254, 271

Mony, B., Kendziorra, E., Maisack, M., Staubert, R., Englhauser, J., Döbereiner, S., Pietsch, W., Reppin, C., Trümper, J., Churazov, E. M., Gilfanov, M. R., \& Sunyaev, R. 1991, A\&A, 247, 405
Nelson, R. W., Vaughan, B. A., Bildsten, L., Finger, M. H., Wilson, R. B., \& Rubin, B. C. 1997, ApJ, 488, L117

Padden, W. E. P., \& Storey, M. C. 1986, PASA, 6, 446

Paul, B., Agrawal, P. C., Rao, A. R., \& Manchanda, R. K. 1997, A\&A, 319, 507

Storey, M. C., Greenhill, J. G., \& Kotani, T. 1998, PASA, $15,217+$

Wang, Y. M., \& Welter, G. L. 1981, A\&A, 102, 97

White, N. E., Swank, J. H., \& Holt, S. S. 1983, ApJ, 270, 711 